\title{
OCCURRENCE OF MICROBIAL FAECAL POLLUTION INDICATORS IN SEDIMENT AND WATER SAMPLES AT ADMIRALTY BAY, KING GEORGE ISLAND, ANTARCTICA
}

http://dx.doi.org/10.4322/apa.2014.043

\author{
Cristina Rossi Nakayama², Priscila Ikeda Ushimaru ${ }^{1}$, Daniela Vilela Lima ${ }^{1}$, Vivian Helena Pellizari1,* \\ ${ }^{1}$ Laboratório de Ecologia Microbiana, Instituto Oceanográfico, Universidade de São Paulo - IOUSP, São Paulo, SP, Brazil \\ ${ }^{2}$ Instituto de Ciências Ambientais, Químicas e Farmacêuticas, Universidade Federal de São Paulo - UNIFESP, Diadema, SP, Brazil \\ *e-mail: vivianp@usp.br
}

Abstract: Assessment of microbial faecal indicators was carried out in water and sediment samples of Admiralty Bay. Quantification of total coliforms, Escherichia coli, sulphite reducing clostridia and Clostridium perfringens was performed through the most probable number technique (MPN) using selective or differential media. Total coliforms were found in all sediment samples, but results may be due to false positive results as a consequence of adaptations in the method to increase its sensitivity. In water, positive results were found in EACF (CF) in all samples along the water column, and in one replicate from Botany Point, Thomas Point and Arctowski. E.coli results were positive only in CF sediment samples and in one Refuge 2 replicate, no E.coli was detected in water. The group of sulphide reducing clostridia showed to be widespread in Martel inlet and was also detected in Refuge 2, suggesting a possible influence of animal faeces in addition to human contribution. C. perfringens was detected in higher numbers in CF and Ullmann Point samples, but positive results were also observed at the other sites. The count values were variable between replicates, but results suggest that contamination in the CF area may persist to a small extent. Analysis of the microbial indicators in samples as animal faeces and a new assessment of the wastewater treatment system are necessary to provide lacking complementary information.

Keywords: microbial faecal indicator, coliforms, clostridia, water, sediment

\section{Introduction}

Traditionally, the determination of faecal coliforms has been used to study the pollution caused by sewage discharge, due to the specificity of the group and the presence of high densities of coliform cells in wastewater. However, the coliforms do not survive long periods under stress in the environment and are less resistant to temperature variations and disinfection processes than some pathogenic microorganisms. For this reason, the quantification of sulphite reducing clostridia (especially Clostridium perfringens) has been used as an auxiliary analysis, since these microorganisms are frequently associated to the gastrointestinal tract of warm-blooded animals and are more resistant to stressful environmental conditions and anoxic conditions in sediments. In general, coliforms and E. coli are indicative of recent contamination and Clostridium perfringens indicates remote contamination (CETESB, 1978). Examples of other Antarctic Stations that assessed faecal indicator microorganisms include the Italian Base at Terra Nova Bay, in Ross Sea (Bruni et al., 1997) and the American MacMurdo Station (Lisle et al., 2004). Both studies were able to detect faecal contamination in areas near sewage discharge using coliform analysis, and at MacMurdo 


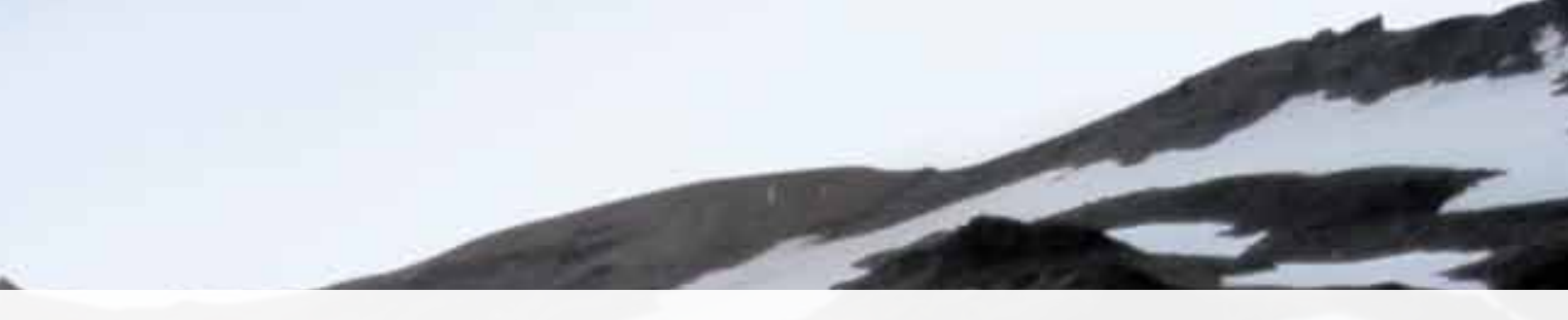

C. perfringens were detected in areas at longer distances from the sewage source. Lisle et al. (2004) also quantified these groups in seal faeces, highlighting that animal source must also be considered.

The present study illustrates the results of faecal pollution indicators from water and sediment collected in the XXVIII Brazilian Antarctic Expedition 2009/2010 through comparison with preterit data obtained in previous studies.

\section{Material and Methods}

\section{Sampling and sample processing}

Water sampling was carried out in February 2010, in five stations at the $30 \mathrm{~m}$ isobaths (Table 1). Samples were collected along the water column at the surface, in the middle and at $1 \mathrm{~m}$ from the bottom, as determined by an ecobathymeter Vexilar mod LPS-1. The samples were stored in borosilicate glass bottles until analysis, which took place in a maximum period of 24 hours after sampling. Samples from all sites and depths were analysed for coliforms. In the case of clostridia, only surface samples were analysed, with exception of CF samples, which received all depth screening.

Sediment sampling was carried out in February-March 2010, at four sites: EACF, and the reference sites Botany Point, Ulmann Point and Refuge 2. For each site, two areas $200 \mathrm{~m}$ distant from each other were sampled in triplicate at 20 to $30 \mathrm{~m}$ depth (Table 2). The areas selected in CF are related to potential pollution sources (the oil tank and the sewage outlet).

Sediment from the $10 \mathrm{~cm}$ top layer was collected aseptically using $60 \mathrm{~mL}$ adapted disposable syringes and stored in sterile whirl pack bags until use. Samples from all sites and replicates were analysed for coliforms. As for Clostridia, analysis of all replicates was carried out at both sites 1 and 2 at EACF and Botany Point and at site 1 at Ulmann Point. Ulmann Point site 2 and Refuge 2 sites 1 and 2 had one triplicate screened only.

\section{Microbiological analysis}

The quantification of coliform and clostridia groups in water and sediment samples was carried out using the Most Probable Number (MPN) technique (APHA, 1995). Coliform and Escherichia coli analysis was performed in the medium Colilert ${ }^{\circledR}$ (IDEXX), which allows the detection of total coliform growth by yellow colour production and E.coli by fluorescence production under UV light. Clostridia were cultivated in media DRCM (differential reinforced clostridial medium) and the presence of $C$. perfringens confirmed by growth in Litmus Milk.

\section{Results and Discussion}

\section{Total coliforms and E. coli}

All sediment samples presented positive counts for total coliforms (Figure 1), with higher values of about $2.5 \times 10^{4} \mathrm{MPN} / 100 \mathrm{~mL}$ being found in CF and BP. These results contrast with previous analysis, in which no coliforms were detected in the reference areas. However, adaptations were made in the sample dilution procedure, based on the protocol adopted by the São Paulo State environmental agency (CETESB) for multiple tubes technique using Lactose Broth, and this change may have increased the probability of occurring false positives, due to

Table 1. Location of water sampling sites in Admiralty Bay

\begin{tabular}{|c|c|c|c|c|}
\hline Station & Name & Latitude (S) & Longitude (W) & Location \\
\hline 1 & Ferraz (CF) & $62^{\circ} 05,216^{\prime}$ & $058^{\circ} 23,175^{\prime}$ & Martel inlet \\
\hline 2 & Botany Point (BP) & $62^{\circ} 05,910^{\prime}$ & $058^{\circ} 20,304^{\prime}$ & Martel inlet \\
\hline 3 & Machu Picchu (MP) & $62^{\circ} 05,357^{\prime}$ & $058^{\circ} 27,558^{\prime}$ & Mackellar inlet \\
\hline 4 & Point Thomas (PT) & $62^{\circ} 09,275^{\prime}$ & $058^{\circ} 29,180^{\prime}$ & Ezcurra inlet \\
\hline 5 & Arctowski (AR) & $62^{\circ} 09,272^{\prime}$ & $058^{\circ} 27,631^{\prime}$ & Admiralty Bay western shore \\
\hline
\end{tabular}




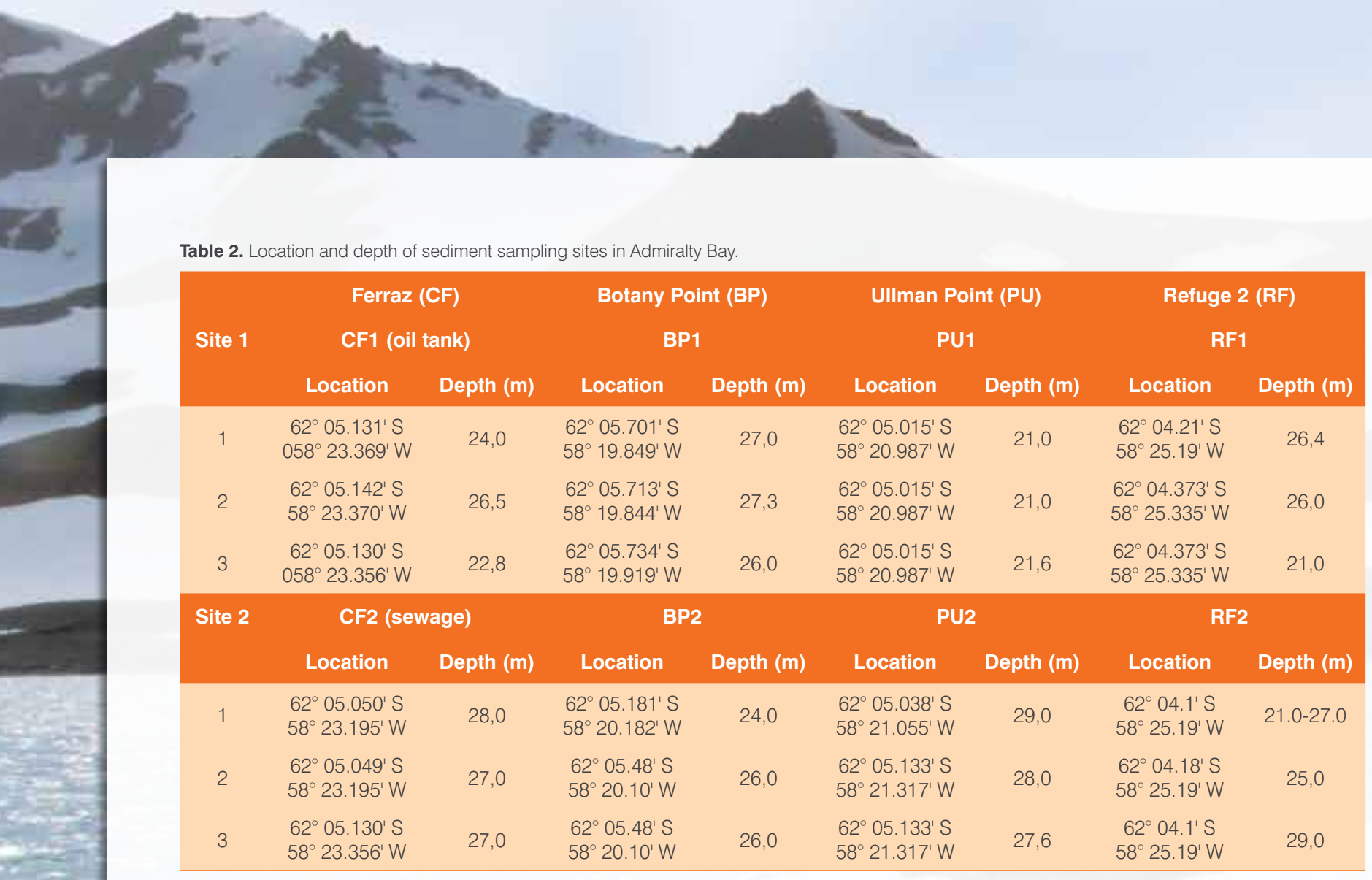

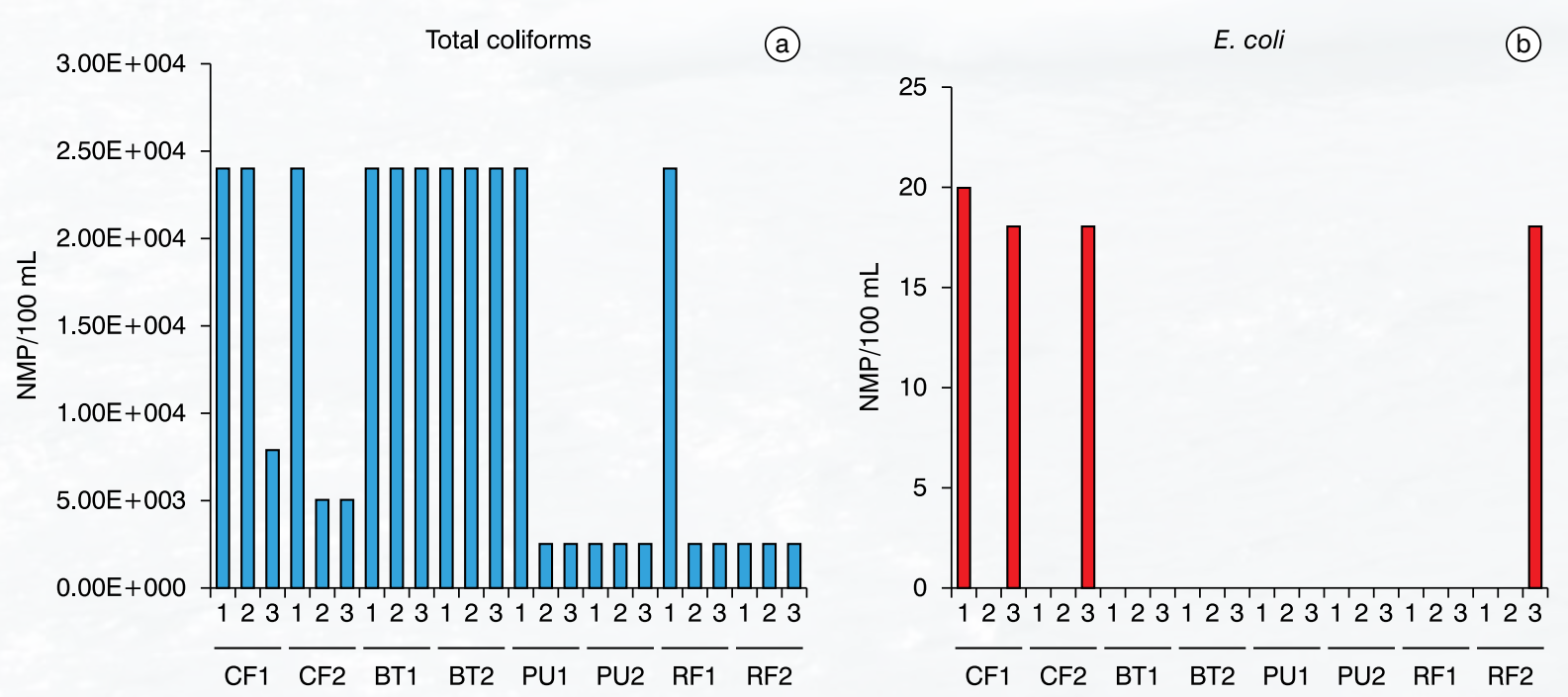

Figure 1. Most probable number counts of total coliforms (a) and E.coli (b) in sediment samples of Admiralty Bay. CF: EACF; BP: Botany Point; PU: Ulmann Point; RF: Refuge 2.

the characteristics of Colilert ${ }^{\oplus}$ medium used in this work. A higher occurrence of false positives in marine samples when using the Colilert ${ }^{\oplus}$ medium was observed by Pisciotta et al. (2002), and it is suggested that higher dilutions be used in order to avoid this bias. In water samples, this massive detection did not occur, but total coliforms were detected in all CF samples along the water column (with values ranging from 13 to $240 \mathrm{MPN} / 100 \mathrm{~mL}$ ), in the bottom sample of BP and PT (13 and $2 \mathrm{MPN} / 100 \mathrm{~mL}$, respectively) and at AT, in the middle water column sample (240 MPN/100 mL). These results may be also related to the contribution of other warm-blooded animals' faeces. 

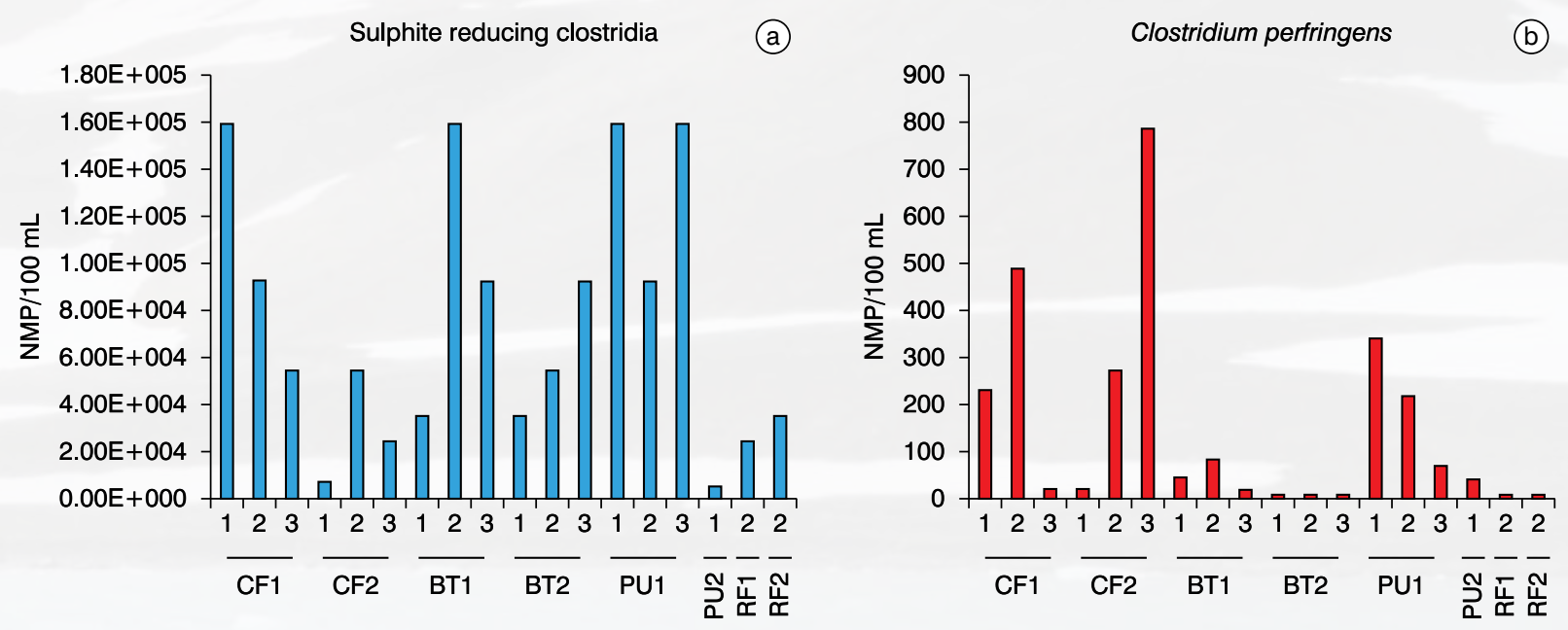

Figure 2. Most probable number counts of sulphite reducing clostridia (a) and C.perfringens (b) in sediment samples of Admiralty Bay. CF: EACF; BT: Botany Point; PU: Ullmann Point; RF: Refuge 2.

In contrast to total coliform results, E. coli analysis in sediment were positive only in two out of three samples in CF1 (oil tank, with values of 18 and $20 \mathrm{MPN} / 100 \mathrm{~mL}$ ) site, in 1 out of 3 samples in CF2 (18 MPN/100 mL), and in 1 of 3 samples of RF (18 MPN/100 mL) (Figure 1). No E. coli was detected in water samples. The values obtained for sediment are lower than the values observed in previous analysis, which revealed counts of up to $790 \mathrm{MPN} / 100 \mathrm{~mL}$ at the CF2 at $20 \mathrm{~m}$ (Montone et al., 2006). As the presence of coliforms is an indicative of recent contamination, this fluctuation may be related to variations in the sewage distribution due to differences in hydrodynamic circulation or even a reduction of the contamination previously detected in the area. Linking up of these results with other parameters is necessary to better analyse the data obtained. Detection of E. coli in one RF sample may also indicate influence of animal faeces.

\section{Sulphite reducing clostridia and C. perfringens}

Both sulphite reducing clostridia and C. perfringens were detected in all sediment samples analysed (Figure 2). Although values varied considerably, no pattern of distribution was observed in Martel inlet, regarding sulphite reducing clostridia, but $\mathrm{CF}$ and $\mathrm{PU}$ were the sites with the highest counts of $C$. perfringens. Low counts were also detected in RF site. Values of C. perfringens in CF sites ranged from 20 to $790 \mathrm{MPN} / 100 \mathrm{~mL}$, but counts did not exceed the values observed previously (800 MPN/100 mL, as described in Montone et al., 2006). No clostridia were detected in water samples.

The results obtained indicate a widespread presence of clostridia in Martel inlet sediment, a behaviour that was also observed in previous studies. The highest values were found at CF sites, but as it is a long term indicator, these results may reflect the influence not only of human activities but also of animal faeces. High variability between counts of replicates also suggests heterogeneity in the distribution of the cells in the sediment.

\section{Conclusions}

The data generated in this study showed high variability of counts between replicates. The detection of higher values of E. coli and C. perfringens in CF samples suggest that contamination in the station area may persist as an impact of small magnitude, and has not increased when comparing present data to previous results. Total coliform analysis may have been subjected to a method bias and future analysis shall consider the use of higher sample dilutions in order to avoid false positive results. Sulphite reducing clostridia was 
